\section{ANAL FISTULA TREATED BY HOIMEOPATHY IN A FRENCH BULLDOG - CASE REPORT}

KEY WORDS: Anal fistula, homeopathy, restoring health

\section{Ana Catarina Viana Valle*}

Aloísio Cunha de Carvalho
Doctor Izao Soares Institute, Integrative Medicine, Ribeirao Preto, Brazil, P.h.D. in Genetics and Biotechnology, UCB - Brasília, Brazil *Corresponding Author

Doctor Izao Soares Institute, Integrative Medicine, Ribeirao Preto, Brazil, P.h.D. in Ambiental Pathology, UNIP - São Paulo, Brazil

Introdution: Anal fistula/perianal is a disease that affects the anal tissue and adjacent skin, associated with severe skin infections. The occurrence of this disease is higher than it appears in the routine of the small animal clinic. The etiology of the perianal fistula has not been elucidated so far. The diagnosis is clinical, and its treatment employs antibiotics, immunosuppressants. However, these medicines are not always efficient, and surgery becomes the only option. Side effects are frequent, and a complementary treatment plan must be evaluated. Homeopathy is a therapeutic technique suggested by the German physician Samuel Hahnemann and aims at healing through stimuli to the body's vital energy. This study aimed to report a perianal fistula occurrence in a dog of the French bulldog breed treated by homeopathy. Methods: Male dog, French bulldog breed, with the complaint of walking and sitting at all times associated with the frequent licking of the anus. After physical examination the diagnosis was perianal fistula. The treatment was based by homeopathy with injectable medicines Belladonna D9 $\left(1 \times 10^{-9}\right)$ associated with Arnica montana D6 $\left(1 \times 10^{-6}\right)$; Nitric acidum $30 \mathrm{CH}\left(1 \times 10^{-60}\right)$; Hamamellis virginiana D18 $\left(1 \times 10^{-18}\right)$. The patient showed clinical improvement right after the first application of the medication, decreasing the stimulus of pain and discomfort. Seventeen days after treatment initiation, the inflammatory process was resolved. Homeopathy has been proposed as a regulatory therapy with a natural tendency to rebalance vital energy and organic functions. The homeopathic medicines were chosen based on the law of similars, i.e., using highly diluted and energized medicines to treat the symptoms and diseases that these same substances, in ponderal doses, would cause in a healthy patient. The treatment here reported did not generate side effects and was effective in its purpose. Treatment time was short, and, additionally, the animal was followed up for eight months after treatment with no relevant clinical changes. Conclusion: The therapeutic protocol determined reestablished the function of the affected tissues with total resolution of the disease in 17 days, with no complications. New comparative and randomized studies must be carried out to better understand the efficacy of such therapy.

\section{INTRODUCTION}

Anal or perianal fistula, also called anal furunculosis, can be characterized as a disease that affects the anal/perianal tissue and adjacent skin. It is usually associated with severe skin infections (Budsberg et al., 1985; Cain, 2011; Ruaux, 2011). The occurrence of this disease is higher than it appears in the routine of the small animal clinic. The etiology of the perianal fistula has not been elucidated so far. However, there are several theories for the occurrence of this problem in dogs. Among the hypotheses, the anatomical location and local contamination are highlighted, which could predispose to a greater degree the occurrence of opportunistic infections (Ellison, 1995; Drager et al., 1998; Cain, 2011; Pieper and McKay, 201 1).In addition, rectal mucus or secretions from anal sacs can accumulate in the perianal region due to possible inflammation from previous injuries to the anal and/or adjacent skin. Furthermore, hypothyroidism or immunodeficiency syndromes may predispose dogs to this disease (Cain, 2011; Ruaux, 2011).

In general, dogs affected by anal fistulas show clinical signs such as excessive licking of the anus and perianal region. This region is sore when the tail is raised, or the area around the anus is touched. Edema, local hyperemia, and ulceration in the latter stage can still beobserved (Pieper and McKay, 2011). Physical examination of the anal region shows, in most dogs, draining wounds and matted fur, along with a fetid odor and moist skin inflammation. The extent and depth of each fistula may vary. Often, excessive effort or discomfort occurs when evacuating, and the appearance of fresh blood can be seen in the feces (Ellison, 1995; Ruaux, 2011).

The diagnosis for anal fistula is clinically made with a complete anamnesis associated with the physical examination. Also, a differential diagnosis should be performed for neoplasms of the affected region or saculitis |www.worldwidejournals.com (anal sac inflammation) (Ellison, 1995; Pieper and McKay, 2011).

Conventional treatment occurs through medications such as antibiotics, immunosuppressants, corticosteroids, and nonsteroidal anti-inflammatory drugs. However, in the literature, it is mentioned that, in general, they have a minimal role in disease regression. Thus, surgery becomes the only option for the complete resolution of the problem (Budsberg et al., 1985; Drager et al., 1998). Fecal incontinence is a potential complication of surgery, especially if there are severe and/or extensive primary lesions. Frequent follow-up is recommended during the first 4-6 weeks of medical therapy because these patients need periodic laboratory tests to monitor the side effects of the medications (Ellison, 1995; Cain, 201 1; Pieper and McKay, 2011).

If the dog responds well to the medical therapy, the prognosis is reasonable to good, but the disease may require lifelong therapy. The prognosis for dogs with more severe disease or a disease requiring surgery and medical therapy is more cautious (uncertain) (Budsberg et al., 1985; Drager et al., 1998). Fistulas can heal and return at a later date. $A$ complementary treatment plan should be evaluated to soften clinical signs and prevent future complications for animals affected by this disease (Dutta, 2019).Homeopathy is a therapeutic technique suggested by the German physician Samuel Hahnemann in 1796 and aims at healing by stimulating the body's vital energy. In other words, this therapy excels in self-healing by only providing stimuli to that energy (Valle et al., 2015). Therefore, several studies are being published in this area, valuing the lowest intervention and maximum organic response of the body in search of its reestablishment (Valle et al., 2020). This study aimed to report the occurrence of a perianal fistula in a dog of the French bulldog breed treated by homeopathy. 


\section{CASE REPORT}

A 4-year-old male dog of the French bulldog breed, neutered and weighing $13 \mathrm{Kg}$, was attended at the NaturalPet Veterinary Clinic in Brasilia, Brazil. The main complaint was walking and sitting at all times associated with the licking of the anus. The patient had normal-colored mucous membranes, adequate hydration conditions, auscultation, heart and respiratory rate within the normal range for age, and physical condition on the physical examination. Absence of abdominal discomfort, temperature $38.5^{\circ} \mathrm{C}$, active, alert. The examination of the anal region showed that it was slightly swollen on the right, hyperemic, and ulcerated. The patient had acute pain on palpation, with no possibility of finishing the local physical examination due to the discomfort generated to the dog. A perianal fistula was then characterized (Fig. 1). Blood was collected for laboratory tests of complete blood count and biochemical measurements of alanine aminotransferase (ALT), alkaline phosphatase (AP), urea, and creatinine. The treatment was instituted by homeopathic therapy, with immediate administration of injectable medicines Belladonna D9 $\left(1 \times 10^{-9}\right)$ (Injectcenter $\left.{ }^{a}\right)$, associated with Arnica montana D6 $\left(1 \times 10^{-6}\right)$ (Injectcenter $\left.{ }^{a}\right)$, in the same syringe, intravenously, for immediate relief. The following medicines were also prescribed: Nitric acidum $30 \mathrm{CH}\left(1 \times 10^{-60}\right)$, alcohol $10 \%$, three drops, twice a day, for 30 days, orally; Belladonna $9\left(1 \times 10^{-9}\right)$ (Injectcenter $\left.{ }^{\hat{a}}\right)$, one ampoule ( $1.1 \mathrm{~mL}$ ), once a day,for ten days, subcutaneously; Arnica montana D6 ( $\left.1 \times 10^{-6}\right)$ (Injectcenter $\left.{ }^{a}\right)$, one ampoule (1.1mL), once a day, for five days, subcutaneously; Hamamellis virginiana D18 $\left(1 \times 10^{-18}\right)$ (Injectcenter ${ }^{\hat{a}}$ ), one ampoule (1.1mL), once a day, for 20 days, subcutaneously.

Fig. 1.(A) Overall Anus Appearance. (B) Ulcerated Perianal Fistula.

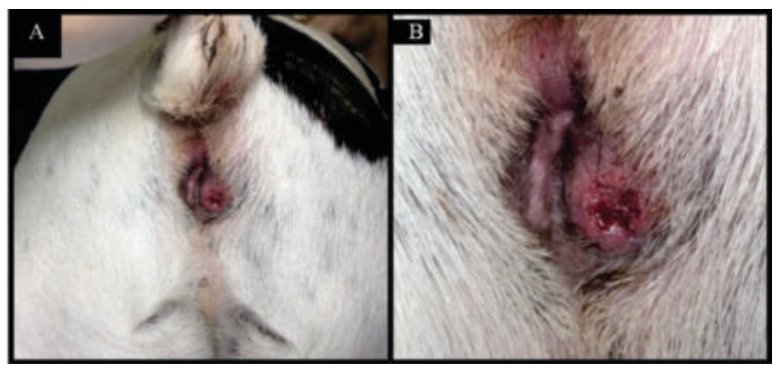

\section{RESULTS AND DISCUSSION}

An anal fistula is a problem that does not have a determined etiology, and its treatment is not well defined. It is predetermined that the clinical treatment based on conventional medicines can cause various long-term side effects. On the other hand, surgical treatment would be the most suitable for complete disease resolution. However, it could cause sequelae to the patient. Given all changes in the animals' lives, such as sequels to the treatments of choice, this article proposed an alternative therapy to the anal/perianal fistula that would not have side effects and would be effective in its purpose.

The patient started to show clinical improvement from the day it was attended at the clinic, after the first medication application. The pain stimulus decreased, as could be inferred by the tutor's declaration that the animal had slept at night, with no sitting and raising all the time, and ate normally. Blood tests resulted in a complete blood count: Red blood cells: 9.470.000/mL; hemoglobin: $21.2 \mathrm{~g} / \mathrm{dL}$; hematocrit: 62.7\%; CMV: 66.21fL; MCHC: $33.81 \mathrm{~g} / \mathrm{dL}$; leukocytes: 7.900/mL; eosinophils: $711 / \mathrm{mL}$; lymphocytes: $1.501 / \mathrm{mL}$; platelets: $219.000 / \mathrm{mL}$; total plasma protein: $6.4 \mathrm{~g} / \mathrm{dL}$; ALT: 46 U/L; creatinine: $1.05 \mathrm{mg} / \mathrm{dL}$; AP: $33 \mathrm{U} / \mathrm{L}$; urea: $34 \mathrm{mg} / \mathrm{dL}$. The patient returned to the clinic for a follow-up visit five days after the first attendance (Fig. 2), and good healing was observed.
Fig. 2. (A) Overall Appearance Of The Anal Region. (B) Healing Of Anal Fistula, Five Days After Treatment Initiation.

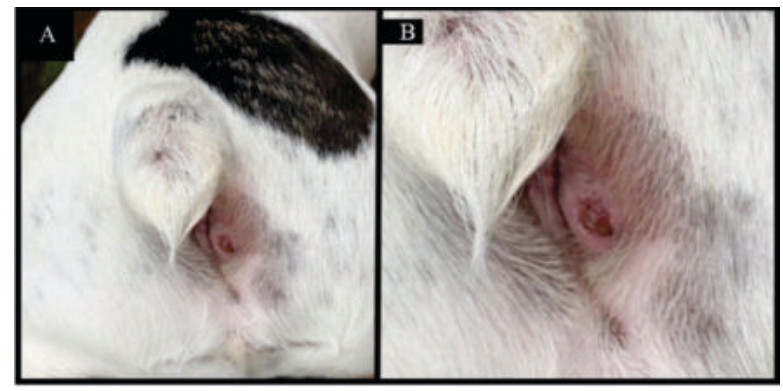

The veterinary follow-up took place every five to seven days until wound healing. The animal was also followed up for eight months after the initial occurrence, with no complications during this period. When the patient returned to the clinic ten days after the first attendance, there was no inflammation in the anal area, and the wound was in the resolution period. No edema or pain as a reaction to local palpation could be observed (Fig. 3). At the end of 17 days, the animal returned to the clinic with no clinical alterations, and the inflammatory process was resolved (Fig. 4).

Fig. 3. (A) Overall Appearance Of The Anal Region. (B) Healing Of Anal Fistula.

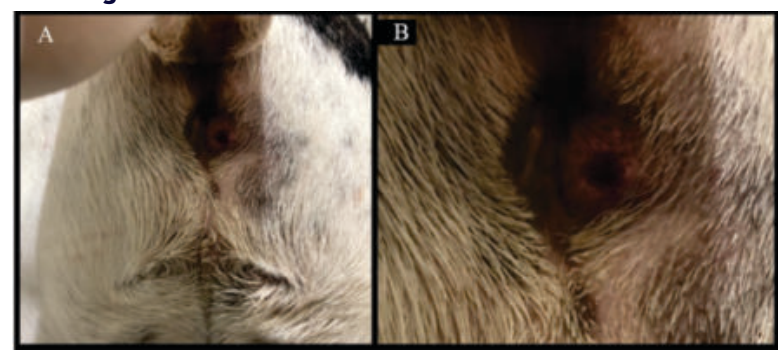

Homeopathy has been studied in several human and animal health areas, such as regulatory therapy, as it uses the body's natural tendency to rebalance vital energy and organic functions (Hahnemann apud Rebollo, 2008). In this context, homeopathic medicines have been prescribed as a therapeutic tool capable of providing significantimprovements in various diseases, minimizing the need for invasive and costly procedures (Jayagopala, 1992). The homeopathic medicines were chosen based on the law of similars, i.e., using highly diluted and energized medicines to treat the symptoms and diseases that these same substances, in ponderal doses, would cause in a healthy patient (Demarque, 2002; Hahnemann apud Rebollo, 2008).

Fig. 4. Overall Appearance Of The Anal Region With Complete Tissue Healing.

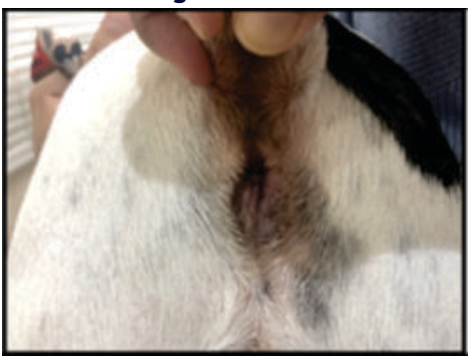

The medicines chosen for the treatment of the reported patient were following the law of anatomopathological similarity. Arnica montana, a plant used for centuries, is indicated in all trauma cases from any source, including in the occurrence of furunculosis. Belladonna, plant originally from Europe and recommended in lesions where there is active congestion with inflammation and acute, sudden, and violent pain. Nitric acidum is chosen in cases of chronic inflammation 
and irritation of the mucous membranes, mainly at the level of the body's orifices, depicting sharp pains, lesions with a tendency to ulceration, and hemorrhages. It is a widely prescribed medication for varicose ulcers, anal fissures, among others. Hamamellis virginiana is a plant originally from North America, containing a large amount of tannin in its structure. It is indicated in cases of bleeding of venous origin, mainly post-traumatic. Hamamellis virginiana is indicated for treating phlebitis, varicose ulcers, and traumatic inflammations on the skin (Cairo, 1991; Vannier e Poirier, 1987).The findings here described are corroborated by Dutta (2019), who reported the treatment of anal fistula following strict homeopathic principles and follow-up at appropriate intervals for about four months. The author describes gradually marked improvement with the medicine Calcarea phosphorica $200 \mathrm{CH}\left(1 \times 10^{-400}\right)$ after completing the course of therapy with excellent results, with no need for more invasive treatments.

The treatment of anal fistulas is complex, and the side effects of the conventional medicines prescribed in clinical therapy, as well as the sequelae caused by surgical procedures, can significantly impact the quality of life of the animals. They can also indirectly impact the lives of their tutors (Ellison, 1995; Drager et al., 1998; Mouatt, 2002; Cain, 2011; Pieper and McKay, 2011; Mathews and Sukhiani, 1997). The treatment reported in this article is of interest to these patients since it did not generate side effects, and it was effective in its purpose. Treatment time was short, and, additionally, the animal was followed up for eight months after treatment with no relevant clinical changes.

\section{CONCLUSION}

The patient presented constant evolution during the prescribed treatment and showed complete healing in 17 days. This data explains the effectiveness of homeopathic therapy when well prescribed. New comparative and randomized studies must be carried out to better understand the efficacy of such therapy.

\section{CONFLICT OF INTEREST}

The authors declare that they have no conflict of interest.

\section{REFERENCES}

1. Budsberg, S.C., Spurgeon, T.L. and Liggitt, H.D. 1985. Anatomic predisposition to perianal fistulae formation in the German shepherd dog. Am J Vet Res. 46(7), 1468-1472.

2. Cain, C.L.2011. Canine Perianal Fistulas: Clinical Presentation, Pathogenesis, and Management.Vet Clin North Am Small Anim Pract. 49(1), 53-65.

3. Cairo, N. 1991. Homeopathic Medicine Guide. Teixeira Press, São Paulo, Brazil.

4. Demarque, D. 2002. Homeopathy: experimental basic medicine. Abrahão Brickman Homeopathy Museum:Ribeirão Preto, SP, Brazil.

5. Drager, L.F., Andrade, M.N.B., Conceição, S.A. and Cunha-Melo, J.R. 1998. Perianal fistula: retrospective study of surgical treatment of 241 cases. Acta Cir.Bras. 13(2),--.doi:https://doi.org/10.1590/\$0102-86501998000200007

6. Dutta, A. 2019. A Case of Anal Fistula Treated by Individualized Homoeopathic Medicine.Innovare J Med Sc. 7(5), 1-3.

7. Ellison, G. 1995. Treatment of perianal fistulas in dogs. J Am Vet Med Assoc. 206, 1680-1682.

8. Hahnemann apud Rebolloco, R.A. Science and metaphysics in Samuel Hahnemann's homeopathy. São Paulo: Philosophical Association Scientiae Studia, 2008

9. Jayagopala, R.N.R. 1992. Homeopathic drug treatment of aural hematomas in dogs. IndianVet J, v.69, p. 1149.

10. Mathews, K.A. and Sukhiani, H.R. 1997. Randomized controlled trial of cyclosporine for treatment of perianal fistulas in dogs. J Am Vet Med Assoc. $211(10), 1249-1253$

11. Mouatt, J.G. 2002. Cyclosporin and ketoconazole interaction for treatment of perianal fistulas in the dog. Aust Vet J.80(4),207-211.

12. Pieper, J. and McKay, L. 2011. Perianal fistulas. Compend Contin Educ Vet. 33(9), E4.

13. Ruaux, C.G. 2011.Perianal Fistula in dogs. by Saunders, Elsevier.

14. Valle, A.C.V., Lima, L., Bonamin, L., Brunel, H., Barros, A., Carvalho, A. and Andrade, R.A. 2020. Use of Viscum album in the Integrative Treatment of Cholangiocarcinoma in a Dog (Canis familiaris) - Case Report. Adv Complement Alt Med. 5(4), 476-481.

15. Valle, A.C.V., Kanaiama, C.Y. and Nader, T. 2015. Homeopathic treatment of aural hematoma in dog: a case report. Unimar Ciências. 24(1-2), 20-26.

16. Vannier, L. and Poirier, J. 1987. Textbook of homeopathic medical matter. (9 ed).Andrei Press: São Paulo, SP, Brazil. 